\title{
A Topic Review: Is Manual Therapy Effective In The Management Of Tension Type Headaches In Primary Care?
}

\author{
Dr Teo Hui Ying Valerie
}

\begin{abstract}
Objective

Headaches are one of the commonest neurological disorders with a global lifetime prevalence of 66 percent (1), and 82.7 percent in Singapore (2). Tension type headache (TTH) is the commonest primary headache with a lifetime prevalence of 30-78 percent globally (I). TTH is related to muscular factors (I7) thus treatment directed toward muscular factors such as manual therapy can be considered (18). This review is aimed at evaluating the use of manual therapy for TTH.
\end{abstract}

\section{Methods}

PubMed and CENTRAL databases were searched in November 2015 with keywords 'tension-type headache' and 'manual therapy' or 'massage' or 'physiotherapy' or 'spinal manipulation' or 'trigger point therapy'. The search was limited to randomised control trials which studied the use of manual therapy in the treatment of TTH.

\section{Results}

8 articles were selected for review. The studies included showed improvement in headache frequency, intensity and Headache Impact Test (HIT-6) scores in favour of manual therapy but not headache duration and Headache Disability Index (global) scores. One study also found an improvement in sick leave days taken.

\section{Conclusion}

Manual therapy reduces headache frequency and intensity of attacks and reduces sick leave days resulting from TTH. There is also a positive effect on the quality of life related to headaches (HIT-6). However, manual therapy does not reduce the duration of a TTH episode. Due to the heterogeneity of manual therapy technique used, it is difficult to recommend a particular regime over another. There is a need for further study of manual therapy as an option for TTH.

Keywords: tension type headache; tension headache; manual therapy; spinal manipulation; massage; physiotherapy;

SFP20 I7; 43(4): 29-34

\section{TEO HUI YING VALERIE}

Family Physician,

Ang Mo Kio Polyclinic

\section{INTRODUCTION}

Headaches are one of the commonest neurological disorders with a global lifetime prevalence of 66 percent, ${ }^{1}$ and 82.7 percent in Singapore. ${ }^{2}$ The majority of patients with headaches are treated in primary care with only a minority referred to specialists. $^{3}$ In 2012, headache was in the top 10 primary diagnoses reported by a Singapore Public Primary Healthcare Group, amounting to 1.8 percent of all clinic visits that year. ${ }^{4}$

The tension-type headache (TTH) is the commonest primary headache with a lifetime prevalence of 30 to 78 percent globally ${ }^{1}$ and is the second most prevalent disorder in the world. ${ }^{5}$ A community-based study conducted in Singapore found that episodic tension-type headache (ETTH) and chronic tension-type headache (CTTH) accounted for 39.9 percent and 2.4 percent of headaches, respectively. ${ }^{2}$

Absenteeism resulting from TTH is considerable, ${ }^{6}$ with a loss of 4.2 workday equivalents per year. ${ }^{7,8}$ TTH also results in repeated emergency department and clinic visits.9,10 Pharmacological treatment includes oral analgesics such as aspirin, acetaminophen, and non-steroidal anti-inflammatory drugs (NSAIDS) in acute attacks. ${ }^{11,12}$ Amitriptyline and mirtazapine are used in the prevention of recurrent TTH. ${ }^{13-15}$

With the rise in chronic diseases such as chronic renal disease ${ }^{16}$ and ischaemic heart disease, adding medications such as NSAIDs and tricyclic antidepressants to a long list of chronic medications may be difficult, thus non-pharmacological alternatives such as manual therapy may be preferred. Some patients have also expressed a preference for alternatives to pharmacological therapy, such as manual therapy. ${ }^{17,18}$

TTH is believed to be related to muscular factors with impairment of the craniocervical musculoskeletal function. ${ }^{19}$ which includes increased tenderness of the pericranial myofascial tissues and myofascial trigger points. Therefore treatment directed toward these factors, such as manual therapy which includes spinal manipulation, trigger point release, massage therapy and physiotherapy may be an effective intervention. ${ }^{20}$ Manual therapy is further defined as skilled passive movement of joints (mobilisation or manipulation) or soft tissue (mobilisation or localised massage). It has been found to have few and usually transient adverse events which include soreness in muscles, stiffness, increased pain, and dizziness. ${ }^{21}$

The use of manual therapy in the treatment of TTH has been studied more extensively in recent years, with randomised controlled trials of improved methodological quality. This topic review thus aims to find out if manual therapy reduces headache frequency, intensity, and headache-related disability for adults with tension-type headaches. 


\section{METHODS}

\section{Literature Search}

Studies were identified by searching electronic databases and the bibliographies of shortlisted articles. A literature search of PUBMED and the Cochrane Central Register of Controlled Trials (CENTRAL) was conducted in November 2015. Manual therapy was defined to include spinal manipulation, massage, trigger point release and physiotherapy but traditional massage and osteopathic treatments, were excluded.

Pubmed was searched using the following strategy: ("tension-type headache" AND "manual therapy"). Various alternative terms to manual therapy such as ("spinal manipulation" OR "massage" OR "trigger point" OR "physiotherapy"). MeSH terms were also used in the search. CENTRAL was searched using: tension headache, manual therapy, massage, trigger point, physiotherapy and combinations of the above. Studies included were limited to randomised control trials in the last 20 years which reported on improvement in headache diary parameters and headache disability indexes in adult patients with the diagnosis of TTH treated with manual therapy. Observational studies, studies involving children, animals and other types of headaches were excluded.

Figure 1 below shows the flowchart of article selection process. A total of 8 full text articles met the inclusion criteria and were analysed for this review. The quality of the articles was assessed using the Jadad score and tabulated in Supplementary Table I $(22-23)$.

Fig. 1: Flow Diagram of Study Selection Process

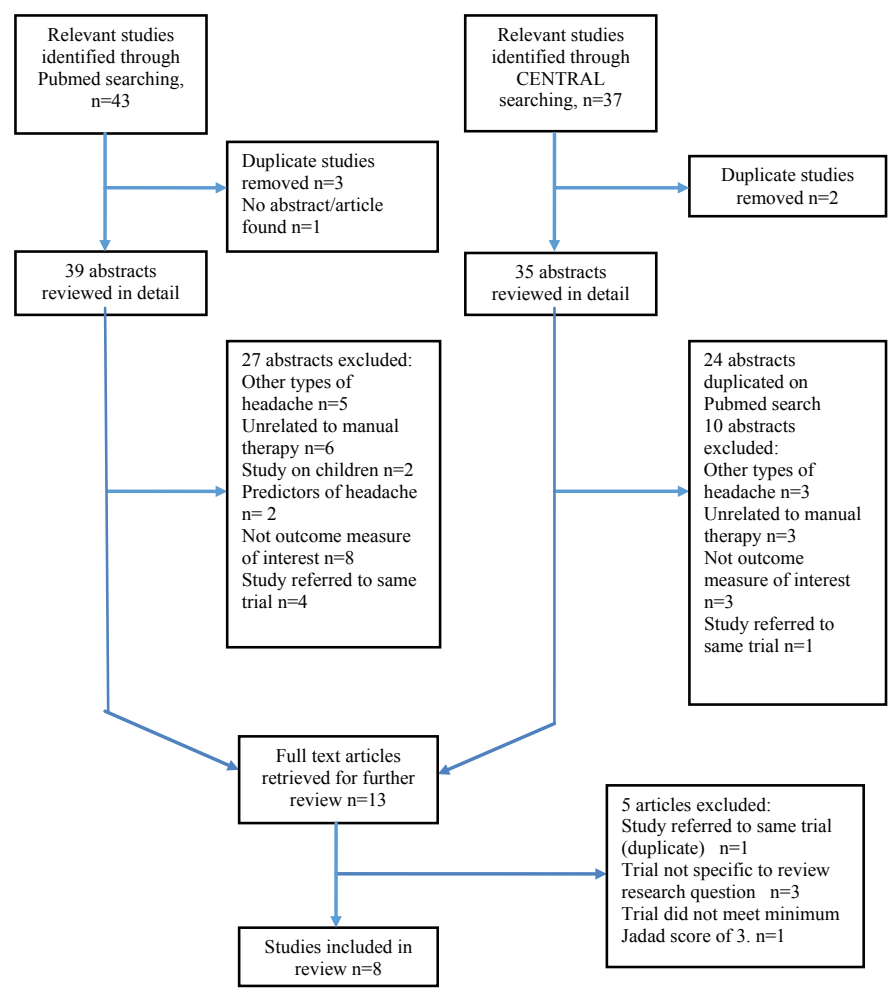

Table I. International Headache Society Guidelines for Tension-type headaches

ETTH is characterised by at least 2 of the following: (a) bilateral location, (b) pressing/tightening quality, (c) mild/moderate intensity and (d) not aggravated by routine physical activity. This should not be associated with nausea or vomiting. Either photophobia or phonophobia may be present.

CTTH is a disorder that evolves from ETTH, with daily or very frequent episodes of headaches lasting minutes to days that occur on at least 15 days per month on average for more than 3 months per year (at least 180 days per year). The headache usually lasts for hours or may be continuous ${ }^{1}$

The following information were extracted from the 8 randomised controlled trials (RCTs) selected: characteristics of the study population; type of TTH, manual therapy method administered; duration of follow up; the placebo/control population; primary and secondary outcomes; and adverse effects.

\section{RESULTS}

All studies had a minimum Jadad score of 3 (details in Supplementary Table I) and studied the use of manual therapy as an intervention for TTH. 6 studies included patients with either CTTH or ETTH and 2 studies only included patients with CTTH. TTH in studies were defined based on the International Headache Society guidelines (details in Table I). Primary outcomes measures included a self-recorded headache diary: frequency of headache, severity and intensity of pain and headache duration.

Other validated questionnaires such as the Headache Impact Test-6 (HIT-6) and Headache Disability Inventory (HDI) with emotional and functional aspects were reported as secondary outcomes. The HIT-6 is a validated questionnaire for assessing quality of life in patients with headache. A decrease of 2.3 to 8 points in this score is considered clinically significant (24-25). The HDI (global) is a measure of headache related disability. A decrease in total HDI of 29 points is considered to be clinically significant (26-27). Other outcomes included sick leave days.

The study characteristics and results are summarised in Table II.

\section{PRIMARY AND SECONDARY OUTCOMES}

\section{Primary outcomes: improvement in headache diary - headache frequency, headache intensity and headache duration}

\section{a. Headache frequency}

Out of 8 randomised controlled trials reviewed, 6 of them (28-29, 31-34) studied the improvement in headache frequency. One study (35) used the headache index- which was a multiplication of headache frequency and intensity. The last study looked at HDI scores as their primary outcome. Different types of manual therapy were applied in the studies.

3 studies involved myofascial trigger point treatment (28, 
31-32) all showed a reduction of headache frequency post therapy. Moraska et al (33) showed significant improvement in headache frequency in the massage group with no significant improvement seen in the control group. Ghanbari et al (31) compared trigger point release therapy (PRT) with usual practice of abortive and prophylactic drugs. Both groups had improved headache frequency after treatment but this improvement was only sustained at follow up in the treatment group. The treatment group showed significant improvement in number of headache days per fortnight from 12.40 to 7.66 days.

Ajimsha et al (32) compared two methods of myofascial release with a control group. The two interventions were direct myofascial release (DT-MFR) and indirect myofascial release (IDT-MFR). Both intervention groups showed significant improvement in number of headache days in 4 weeks ranging from 6.7 to 7.1 days in both treatment groups but an improvement of only 1.6 days in the placebo group. 81.6 percent to 86.4 percent of patients in the treatment groups reported at least 50 percent reduction in headache days compared to baseline, whereas the placebo group had none.

Three studies used spinal manipulation techniques $(29,33,35)$. Espí-López et al (29) studied 2 manual therapy treatments, sub-occipital soft tissue inhibition (SI) and occiput-atlas-axis (OAA) articulatory manipulation. Combination therapy showed significant improvement in headache frequency. This was sustained at 4 week follow up. The effect size was calculated to be 1.15. The OAA group also had significant improvement in headache frequency post treatment; however, this was not sustained at follow up. Both SI and control groups did not have significant improvements in headache frequency.

Castien et al (33) compared manual therapy involving mobilisations of the cervical and thoracic spine against usual GP care (Dutch general practice guideline for headache management). Manual therapy decreased headache days per fortnight by $9.1 \pm 3.8$ days, compared to $2.7 \pm 4.3$ days with usual care. The mean difference was significant in favour of manual therapy. The manual therapy group showed sustained improvement of $9.1 \pm 4.2$ days at 6 month follow up. 81.6 percent of participants in manual therapy group and 40.5 percent in usual care group had at least 50 percent reduction in headache frequency at 6 months. The number needed to treat was 3 with an effect size of 1.2 .

Demirturk et al (35) compared 2 methods of manual therapy, connective tissue manipulation (CTM) and Dr. James Cyriax's vertebral mobilisation. The measured outcome was headache index (HI), which was headache frequency multiplied by pain severity. Both groups showed significant improvements in $\mathrm{HI}$ from baseline but difference between groups was not significant. In all patients except one, HI values were lower than baseline.

Ettenkoven et al (34) compared physiotherapy against a combined physiotherapy programme with a craniocervical training (CTP); there was no placebo group for comparison. Both groups had significantly reduced headache frequency after treatment, but only the CTP group had further reduction at 6 months follow up. Details of results are seen below in Supplementary Table II.

\section{b. Headache intensity}

Headache intensity was reported in 5 studies (28-29, 31, 33-34). The studies involving myofascial trigger point therapy $(28,31)$ did not show any significant improvement in headache intensity compared to the control groups.

Both studies (29, 33) involving spinal manipulation showed improvements in headache intensity. EspI-López et al (29) showed improvement in headache intensity (based on a $0-10$ visual analogue scale) in the combination therapy group, OAA group and control group. However, the combination therapy group had the greatest improvement and an effect size of 1.15 . The OAA group had the second largest effect size of 0.91 with an improvement from $5.12 \pm 1.95$ to $3.03 \pm 2.80$ post treatment. Both groups had sustained improvements at follow up.

Castien et al (33) found significant improvement in headache intensity favouring manual therapy. This was sustained at 6 month follow up. Manual therapy group had improved scores on a 0 to 10 numeric rating scale of $2.7 \pm 0.9$ points compared to 0.9 \pm 2.4 points in usual GP care group. The improvement in headache intensity at 6 month follow up similarly was in favour of manual therapy. Ettenkoven et al (34) reported improvement in headache intensity in both physiotherapy groups; however the improvement was only sustained on follow up in the physiotherapy group with CTP. The results are summarised below in Supplementary Table III.

\section{c. Headache duration}

The studies showed mixed results for headache duration; only 2 studies showing improvement in headache duration $(31,33)$. Ghanbari et al (31) showed that the intervention group receiving trigger point release therapy had an improvement in headache duration from $6.42 \pm 5.70$ hours to $3.84 \pm 4.35$ hours post treatment. There was further improvement at 2 -week follow up for the therapy group. There was no significant improvement in headache duration in the control group.

Castien et al (33) found an improvement in headache duration in favour of the manual therapy of $5.9 \pm 8.7$ hours per day compared to $0.60 \pm 10.0$ hours with usual care. However, there was no statistical significance between groups at 6 month follow up. The results are summarised below in Supplementary Table IV.

\section{Secondary Outcomes: Improvement in HDI (global) Scores and HIT-6 Scores}

\section{a. HDI (global) scores}

HDI (global) scores were measured in 4 studies $(28-30,33)$ and showed improvements but were not clinically significance.

\section{b. HIT-6 scores}

HIT-6 scores were measured in 3 studies $(28-29,33)$ and consistently showed improvement in favour of manual therapy and was clinically significant. Moraska et al (28) showed improvement in HIT-6 scores for both massage and placebo groups but no significant improvement in the control group. The massage group had a 
larger improvement from $56.3 \pm 1.57$ to $50.2 \pm 1.57$ points which was both statistically and clinically significant. Espí-López et al (29) found improved HIT-6 scores for all intervention groups but not in the control group. HIT-6 scores had the largest improvement in the OAA group, from $60.23 \pm 5.85$ points to $53.50 \pm 6.32$ points post treatment and $52.85 \pm 6.27$ points at follow up. The combination therapy group had the second largest improvement in HIT-6 scores and an effect size of 0.93. Castien et al (33) showed improvement in HIT-6 scores of $8.9 \pm 7.1$ points with manual therapy compared to $2.4 \pm 6.5$ points with usual care. This improvement was sustained at 6 months.

\section{OTHER OUTCOME MEASURES}

\section{Sick Leave Days Taken Due to Headache}

Castien et al (33) found that 7.9 percent with manual therapy compared to 32.4 percent with usual care reporting at least one day of sick leave during the 6-month follow up period. This was statistically significant.

\section{Adverse Events}

There was only one reported adverse (29) reported but no details were reported.

\section{DISCUSSION}

\section{Summary of Findings}

In this review, manual therapy had shown (with varying techniques) to decrease frequency of headache days during acute attacks and appears to have sustained benefit at follow up which is non-inferior to and in several studies more effective than pharmacological therapy. There was also improvement in headache intensity reported in 6 of the studies reviewed. The HIT-6 scores improved in favour of manual therapy; this was used to assess the quality of life of patients with CTTH or ETTH. However, manual therapy did not shorten the duration of a TTH episode. There was also reported reduction in sick leave days in the 6 months after intervention. This however was only measured in one study (33).

Reviews that were previously done on the effectiveness of manual therapy in the treatment of TTH found a lack of rigorous evidence that manual therapy improved pain outcomes in patients with TTH (36-37). A review on spinal manipulation had shown encouraging evidence in treating TTH but was inconclusive due to low quality of studies reviewed (38). The recent studies have improved methodologies. As double blinding was difficult due to the nature of the interventions, strict protocols and blinding of assessors was used to improve the rigor.

The modalities of manual therapy used varied between studies. The studies focusing on spinal manipulation provided the most consistent results with improvement in headache frequency, head intensity and also reduction in sick leave days reported by Castien et al (33). The studies involving myofascial trigger point therapy had improvement in headache frequency and HIT-6 scores; however, had no improvement in headache intensity and duration. Only one study looked at physiotherapy; however, this was done by comparing two different therapy techniques. Therefore evaluation of effectiveness compared to medications or placebo was not possible.

In view of the variety and heterogeneity of manual therapy techniques available, there have been attempts to identify the techniques of manual therapy that are effective in the treatment of TTH. Fernandez et al (39) suggests an approach that includes inactivation of actives trigger points in the muscles of the head and neck regions such as the trapezius and temporalis muscles, cervical mobilisation/manipulation and exercises targeted at neck flexion and extension. Therefore, the manual therapy of choice may be a combination of techniques rather than one particular technique.

\section{Clinical Implications}

This review has shown that manual therapy is effective in reducing headache frequency, intensity and improving quality of life related to TTH. Majority of headaches are treated in primary care and primary care physicians may consider manual therapy as a possible modality of treatment for TTH $(40-41)$. This is especially so in patients who may not be suitable for pharmacotherapy or those who have a preference for non-pharmacological options.

However, physicians must be aware of the possible complications of manual therapy so as to appropriately advice their patients. They must also conduct proper physical examinations to exclude contraindications and red flags so as to minimise adverse events $(42)$.

\section{Limitations}

The limitations of the studies reviewed include recruitment bias. In majority of the studies, there were a greater proportion of female patients recruited and one trial only included female patients. This thus affects the applicability of the results to male patients with TTH.

There is significant heterogeneity of the interventions in the studies reviewed, with varying techniques of manual therapy used. The duration of treatment and follow up also varied with some studies looking at short term effects during 2 to 4 week follow ups and other studies looking at more long term benefits with follow up after 6 months. This limited direct comparison between techniques and comparison to pharmacotherapy.

Another limitation is the failure of adequate blinding in all but three of the studies reviewed. Due to the nature of the intervention it was difficult for double blinding. However, some studies had made additional attempts at reducing bias in the study through strict protocols and strict blinding of assessors of the study.

\section{Future Research}

There is a need for future research in this area. In future studies, there may need to be standardisation of manual therapy method 
used to achieve a more consistent effect and results. There should also be standardisation of the outcome measures looking at headache diary: headache frequency, intensity, duration and also headache impact scores such as HDI and HIT-6.

There was only one study which reported the reduction in sick days taken, this would be a good index of measure for the effectiveness of therapy that can be considered in future research. It is known that TTH leads to considerable absenteeism and in turn has a socioeconomic impact.

The adverse effects of manual therapy have not been well described in these clinical trials, even though the studies reported the occurrence of adverse events. Manual therapy has been known to cause stiffness, increased pain, limitation in motion in some patients and more uncommon but serious injuries such as neurological deficits and carotid dissection (21, 43-44). In order to understand the impact of manual therapy better, more studies on its adverse effects are necessary.

Lastly, future research to compare different modalities of treatment for TTH directly will be useful as well. This would allow physicians to choose between different modalities of manual therapy or combinations of manual therapy techniques.

\section{CONCLUSION}

This review shows that manual therapy reduces headache frequency and intensity of attacks and reduces sick leave days resulting from TTH. There is also a positive effect on the quality of life related to headaches (HIT-6). However, manual therapy does not reduce the duration of a TTH episode. Therefore, it is a possible treatment modality for TTH that may be considered for patients who are unable to tolerate pharmacotherapy or may prefer a non-pharmacological treatment. However, it is difficult to recommend a particular type of manual therapy due to the heterogeneity of techniques used.

There is a need for further study of manual therapy as an option for TTH with a focus on outcomes such as headache diary, quality of life related to headaches, sick leave days taken as a result of TTH and also the adverse events related to it.

\section{REFERENCES}

I. Olsen J, Bousser M-G, Diener, H-C, Dodick D, First M, Goadsby PJ, et al. The International Classification of Headache Disorders 2nd edition [Internet]. International Headache Society; 2004 [cited 2016 Jan 16]. Available from:

http://ihs-classification.org/_downloads/mixed/WatermarkedShortForm \%20ICHD-II.pdf.

2. Ho KH, Ong BK. A community-based study of headache diagnosis and prevalence in Singapore. Cephalalgia 2003 Feb;23(I):6-13.

3. Laughrey WF, Holmes WF, Macgregor AE, Sawyer JPC. Headache consultation and referral patterns in one UK general practice.

Cephalagia 1999; 19:328-329.

4. Lai PC, editor. National Healthcare Group Polyclinics Annual Report FY20I2 [Internet]. National Healthcare Group Polyclinics [cited 2016

Apr 28]. Available from:

https://www.nhgp.com.sg/uploadedFiles/NHGP\%20AR\%20FY20I2_We bsite.pdf.
5. Martelletti P, Birbeck GL, Katsarava Z, Jensen RH, Stovner LJ, Steiner TJ. The Global Burden of Disease survey 20I0, Lifting The Burden and thinking outside-the-box on headache disorders. J Headache Pain. 2013;14(1):13.

6. Chowdhury D. Tension type headache. Ann Indian Acad Neurol. 2012 Aug; I5(SupplI): S83-S88.

7. Lyngberg AC, Rasmussen BK, Jorgensen T, Jensen R. Secular changes in health care utilization and work absence for migraine and tension-type headache: A population based study. Eur J Epidemiol. 2005;20(I2): 1007-I4.

8. Schwartz BS, Stewart WF, Lipton RB. Lost workdays and decreased work effectiveness associated with headache in the workplace. J Occup Environ Med. 1997 Apr;39(4):320-7.

9. Stovner LJ, Hagen K, Jensen R, Katsarava Z, Lipton RB, Scher Al, et al. The global burden of headache: a documentation of headache prevalence and disability worldwide. Cephalalgia. 2007 Mar;27(3):193-210.

10. Vos T, Flaxman AD, Naghavi M, Lozano R, Michaud C, Ezzati M, et al. Years lived with disability (YLDs) for I 160 sequelae of 289 diseases and injuries 1990-2010: a systematic analysis for the Global Burden of Disease Study 2010. Lancet. 20I 2 Dec I5; 380(9859):2I63-96.

I I. Steiner TJ, Lange R, Voelker M. Aspirin in episodic tension-type headache: Placebo-controlled dose-ranging comparison with paracetamol. Cephalalgia. 2003 Feb;23(I):59-66.

12. Ashina S, Ashina M. Current and potential future drug therapies for tension-type headache. Curr Pain Headcahe Rep. 2003

Dec;7(6):466-74.

13. Lance JW, Curran DA. Treatment of chronic tension headache. Lancet. 1964 Jun; I (7345): I236-9.

14. Gobel H, Hamouz V, Hansen C, Heininger K, Hirsch S, Lindner V, et al. Chronic tension-type headache: amitriptyline reduces clinical headache-duration and experimental pain sensitivity but does not alter pericranial muscle activity readings. Pain. 1994 Nov; 59(2):24I-9.

15. Bendtsen L, Jensen R. Mirtazapine is effective in the prophylactic treatment of chronic tension type headache. Neurology. 2004 May;62(I0): 1706-II.

16. Chong HL, Anantharaman V, editors. Singapore Renal Registry Report No. 10. Trends in Chronic Kidney Failure Stage 5 in Singapore 2012/2013 [Internet]. National Registry of Diseases Office [cited 2016 Apr 15]. Available from:

https://www.nrdo.gov.sg/docs/librariesprovider3/Publications---KidneyFailure/trends-in-chronic-kidney-failure-stage-5-in-singapore.pdf?sfvrsn= 0\&AspxAutoDetectCookieSupport=I

17. Castien R, van der Windt DAWM, Dekker J, Mutsaers B, Grooten A. Effectiveness of manual therapy compared to usual care by the general practitioner for chronic tension-type headache: design of a randomised clinical trial. BMC Musculoskeletal Disorders [Internet]. 2009 [cited 2016 Mar 12]; 10:21. Available from:

http://bmcmusculoskeletdisord.biomedcentral.com/articles/ / 0.1 I86//47 |-2474-|0-2|.

18. Rossi P, Di Lorenzo G, Faroni J, Malpezzi MG, Cesarino F, Nappi G. Use of complementary and alternative medicine by patients with chronic tension-type headache: results of a headache clinic survey. Headache. 2006 Apr;46(4):622-3I.

19. Fumal A, Schoenen J. Tension-type headache: current research and clinical management. Lancet Neurol. 2008 Jan;7(I):70-83.

20. Bendtsen $L$, Fernández-de-las-Peñas $C$. The role of muscles in tension-type headache. Curr Pain Headache Rep. 2011

Dec; I5(6):45।-8.

2I. Carnes D, Mars TS, Mullinger B, Froud R, Underwood M. Adverse events and manual therapy: a systematic review. Man Ther. 2010 
Aug;15(4):355-363.

22. Jadad AR, Moore RA, Carroll D, Jenkinson C, Reynolds DJ, Gavaghan DJ, et al. Assessing the Quality of Reports of Randomized Clinical Trials: Is Blinding Necessary? Controlled Clinical Trials. 1996 Feb; $17(1): 1-12$.

23. Berger VW, Alperson SY. A General Framework for the Evaluation of Clinical Trial Quality. Rev Recent Clin Trials. 2009 May;4(2):79-88.

24. Coeytaux RR, Kaufman JS, Chao R, Mann JD, Devellis RF. Four methods of estimating the Headache Impact Test. Journal of Clinical Epidemiology. 2006 Apr;59(4):374-80.

25. Castien RF, Blankenstein AH, Windt DA, Dekker J. Minimal clinically important change on the Headache Impact Test-6 questionnaire in patients with chronic tension-type headache. Cephalalgia. 2012 Jul;32(9):7I0-4.

26. Jacobson GP, Ramadan NM, Aggarwal SK, Newman CW. The Henry Ford Hospital Headache Disability Inventory (HDI). Neurology. 1994 May; 44(5):837-42.

27. Jacobson GP, Ramadan NM, Norris L, Newman CW. Headache Disability Inventory (HDI): short-term test-retest reliability and spouse perceptions. Headache. 1995 Oct; 35(9):534-9.

28. Moraska AF, Stenerson L, Butryn N, Krutsch JP, Schmiege SJ, Mann JD. Myofascial trigger point-focused head and neck massage for recurrent tension-type headache: A randomized, placebo-controlled clinical trial. Clin J Pain. 2015 Feb;3 I(2):I59-68.

29. Espī-López GV, Gómez-Conesa A, Gómez AA, Martínez JB, Pascual-Vaca AO, Blanco CR. Treatment of tension-type headache with articulatory and suboccipital soft tissue therapy: A double-blind, randomized placebo-controlled clinical trial. J Bodyw Mov Ther. 2014 Oct; |8(4):576-85.

30. Espí-López GV, Rodríguez-Blanco C, Oliva-Pascual-Vaca A, Benítez-Martínez JC, Lluch E, Falla D. Effect of manual therapy techniques on headache disability in patients with tension-type headache. Randomized controlled trial. Eur J Phys Rehabil Med. 2014 Dec;50(6):64I-7.

3I. Ghanbari A, Rahimijaberi A, Mohamadi M, Abbasi L, Sarvestani FK. The effect of trigger point management by positional release therapy on tension type headache. NeuroRehabilitation. 2012;30(4):333-9.

32. Ajimsha MS. Effectiveness of direct vs indirect technique myofascial release in the management of tension-type headache. J Bodyw Mov Ther. 20II Oct; I5(4):43I-5.

33. Castien RF, van der Windt DA, Grooten A, Dekker J. Effectiveness of manual therapy for chronic tension-type headache: A pragmatic, randomised, clinical trial. Cephalalgia. 20I I Jan;3I (2):I33-43.

34. van Ettekoven $\mathrm{H}$, Lucas $\mathrm{C}$. Efficacy of physiotherapy including a craniocervical training programme for tension-type headache; a randomized clinical trial. Cephalalgia. 2006 Aug;26(8):983-91.

35. Demirturk F, Akarcali I, Akbayrak T, Citak I, Inan L. Results of two different manual therapy techniques in chronic tension-type headache. The Pain Clinic. 2002; 14(2): I 2 I-8.

36. Lenssinck M-LB, Damen L, Verhagen AP, Berger MY, Passchier J, Koes BW. The effectiveness of physiotherapy and manipulation in patients with tension-type headache: a systematic review. Pain. 2004 Dec; I I2(3):38I-388.

37. Fernández-de-las-Peñas C, Alonso-Blanco C, Cuadrado ML, Miangolarra JC, Barriga FJ, Pareja JA. Are Manual Therapies Effective in Reducing Pain From tension-Type Headache? A systematic review. Clin J Pain. 2006 Mar-Apr;22(3):278-85.

38. Posadzki P, Ernst E. Spinal manipulations for tension-type headaches: A systematic review of randomized controlled trials. Complimentary Therapies in Medicine. 2012 Aug; 20(4):232-9.

39. Fernández-de-las-Peñas C, Courtney CA. Clinical reasoning for manual therapy management of tension type and cervicogenic headache. J Man Manip Ther. 20I4 Feb; 22(I):44-50.

40. Bendtsen L, Evers S, Linde M, Mitsikostas DD, Sandrini G, Schoenen J; EFNS. EFNS guideline on the treatment of tension-type headache - report of an EFNS task force. Eur J Neurol. 2010 Nov; I7(II):I318-25.

4I. Millea PJ, Brodie JJ. Tension-Type Headache. Am Fam Physician. 2002 Sep I;66(5):797-805.

42. Puentedura EJ, March J, Anders J, Perez A, Landers MR, Wallmann HW, et al. Safety of cervical spine manipulation: are adverse events preventable and are manipulations being performed appropriately?: A review of I34 case reports. J Man Manio Ther. 20I2 May;20(2):66-74. 43. Cagnie B, Vinck E, Beernaert A, Cambier D. How common are side effects of spinal manipulation and can these side effects be predicted? Man Ther. 2004 Aug;9(3): I5I-6.

44. Ernst E. Manipulation of the cervical spine: a systematic review of case reports of serious adverse events, 1995-200I.Med J Aust. 2002 Apr;176(8):376-80. 